\title{
Low-dose theophylline enhances the anti- inflammatory effects of steroids during exacerbations of COPD
}

\author{
B G Cosio, ${ }^{1,2,3}$ A Iglesias, ${ }^{2}$ A Rios, ${ }^{3}$ A Noguera,,${ }^{1,2,3}$ E Sala, ${ }^{1,2,3} \mathrm{~K}$ Ito, ${ }^{4}$ P J Barnes, ${ }^{4}$ \\ A Agusti, 1,3
}

${ }^{1}$ Department of Respiratory Medicine, Hospital Universitario Son Dureta, Palma de Mallorca, Spain: ${ }^{2}$ CIBER de Enfermedades Respiratorias, Unidad de Investigacion, Hospital Son Dureta, Palma de Mallorca, Spain; ${ }^{3}$ Epidemiology and Clinical Research Unit, Fundacion Caubet-Cimera, Bunyola, Spain; ${ }^{4}$ Airway Disease Section, National Heart and Lung Institute, Imperial College, London, UK

Correspondence to: Dr B G Cosío, Department of Respiratory Medicine, Hospital Universitario Son Dureta, Andrea Doria 55, 07014 Palma de

Mallorca, Spain; borja.cosio@ ssib.es

Received 16 June 2008 Accepted 8 January 2009 Published Online First 20 January 2009
ABSTRACT

Background: Chronic obstructive pulmonary disease (COPD) is characterised by an abnormal inflammatory response mainly to cigarette smoke that flares up during exacerbations of the disease (ECOPD). Reduced activity of histone deacetylases (HDAC) contributes to enhanced inflammation in stable COPD. It was hypothesised that HDAC activity is further reduced during ECOPD and that theophylline, an HDAC activator, potentiates the antiinflammatory effect of steroids in these patients. A study was performed to investigate HDAC activity during ECOPD and the effects of theophylline on the anti-inflammatory effects of steroids in a randomised single-blind controlled study.

Methods: 35 patients hospitalised with ECOPD and treated according to international guidelines (including systemic steroids) were randomised to receive or not to receive low-dose oral theophylline (100 mg twice daily). Before treatment and 3 months after discharge, HDAC and nuclear factor- $\kappa \mathrm{B}$ (NF- $\kappa \mathrm{B}$ ) activity in sputum macrophages, the concentration of nitric oxide in exhaled air (eNO) and total antioxidant status (TAS), tumour necrosis factor $\alpha$ (TNF $\alpha$ ), interleukin (IL)-6 and IL8 levels in sputum supernatants were measured.

Results: Patients receiving standard therapy showed decreased NF- $\kappa$ B activity, eNO concentration and sputum levels of TNF $\alpha$, IL6 and IL8, as well as increased TAS during recovery of ECOPD, but HDAC activity did not change. The addition of low-dose theophylline increased HDAC activity and further reduced IL8 and TNFa concentrations.

Conclusions: During ECOPD, low-dose theophylline increases HDAC activity and improves the anti-inflammatory effects of steroids.

Trial registration number: NCT00671151

Chronic obstructive pulmonary disease (COPD) is characterised by an enhanced inflammatory response to inhaled particles and gases, mostly cigarette smoking. ${ }^{1}$ We have previously shown that the activity of histone deacetylases (HDAC) is decreased in patients with stable COPD, and that this can contribute to the abnormal inflammatory response that characterises the disease. ${ }^{23}$

Patients with COPD often experience acute episodes of exacerbation (ECOPD) during the course of their disease. These episodes are characterised by nuclear factor- $\kappa \mathrm{B}(\mathrm{NF}-\kappa \mathrm{B})$ activation $^{4}{ }^{5}$ and a burst of airway inflammation, with increased oxidative and nitrative stress, ${ }^{67}$ increased neutrophil counts and raised levels of several proinflammatory cytokines such as tumour necrosis factor $\alpha($ TNF $\alpha)$ and interleukin-8 (IL8) and IL6..$^{8-10}$ Because both oxidative $^{11}$ and nitrative stress ${ }^{12}$ can impair HDAC function, we hypothesised that HDAC activity will be further reduced during ECOPD, thus contributing to amplify baseline inflammation.

Current international guidelines recommend the use of high-dose oral or intravenous steroids (in combination with inhaled bronchodilators) for the treatment of ECOPD. ${ }^{1}$ Low concentrations of theophylline enhance HDAC activity and have anti-inflammatory effects, both in asthma and COPD, ${ }^{13-15}$ that have been shown to add clinical benefits and safety in the long-term treatment of COPD. ${ }^{16}$

We have previously shown that an impaired HDAC activity reduces the anti-inflammatory effects of steroids in patients with stable $\mathrm{COPD},{ }^{23}$ and that this can be reversed by lowdose theophylline. ${ }^{17}$ We hypothesised here that theophylline can also enhance HDAC activity and improve steroid responsiveness during ECOPD.

Accordingly, in this prospective randomised single-blind controlled study, we determined as the primary outcome the activity of HDAC in sputum macrophages of patients hospitalised with ECOPD and investigated whether the addition of low-dose oral theophylline to standard therapy enhances its activity and the anti-inflammatory effects of steroids.

\section{METHODS \\ Patients}

Patients were recruited within the first $24 \mathrm{~h}$ of admission from the Accident and Emergency department of Hospital Universitario Son Dureta from June 2005 to December 2007. Subjects were eligible if they had COPD, defined as a postbronchodilator forced expiratory volume in $1 \mathrm{~s} /$ forced vital capacity ( $\left.\mathrm{FEV}_{1} / \mathrm{FVC}\right)$ ratio of $<70 \%$ determined at stability according to GOLD criteria, ${ }^{1}$ a smoking history of $>15$ pack-years and a diagnosis on admission of ECOPD. The latter was made by the emergency physician who was unaware of the study, and was based on the patient's symptoms and complementary tests-namely, arterial blood gases, ECG, chest radiography and routine blood tests. Patients with a history of asthma, bronchiectasis, carcinoma of the bronchus, pneumonia or heart failure were excluded. Patients were also excluded if they were unable to provide an adequate sputum sample or if they were already being treated with theophylline, anti-inflammatory therapy for chronic inflammatory diseases such as Crohn's 
disease or rheumatoid arthritis, or had received antibiotics or oral steroids during the previous 4 weeks. Co-morbidities were assessed by the Charlson co-morbidity index.

\section{Study design}

On admission all patients received treatment according to international guidelines ${ }^{1}$ that included nebulised bronchodilators ( $\beta$ agonists and anticholinergics), systemic steroids (oral or intravenously) in all patients with or without antibiotics according to Anthonisen's criteria. ${ }^{18}$ When the patient accepted to participate in the study, spirometry, arterial blood gases, exhaled nitric oxide (eNO) measurements and induced sputum samples were obtained during the first $24 \mathrm{~h}$ of hospitalisation. Patients were then randomised to receive standard therapy with or without theophylline (100 mg twice daily) that was maintained until the end of the study. At discharge, both groups received treatment with inhaled corticosteroids, longacting $\beta$ agonist and long-acting anticholinergic agents. Randomisation was made by a research nurse on the basis of a computer-generated randomisation list and the investigators who analysed the data were unaware of the treatment arms.

An outpatient clinic visit was scheduled 3 months after discharge. Clinical stability was accepted if the patient had not needed to change his regular treatment. If another ECOPD episode occurred after discharge, patients were rescheduled 3 months later. During this outpatient visit, measurements obtained during hospitalisation were repeated. If the patient did not attend the visit, the investigator team contacted the patient to confirm survival.

\section{Lung function}

Forced spirometry (GS, Warren E Collins, Braintree, Massachusetts, USA) was obtained in all participants according to international guidelines. ${ }^{19}$ Spirometric reference values were those of a Mediterranean population. ${ }^{20}$ Previous lung function was used to confirm the diagnosis of COPD, when available $(n=39)$.

\section{Exhaled nitric oxide measurement}

The measurement of eNO was performed with a chemiluminescence analyser (Sievers Instruments Inc, Model 280NOA, Boulder, Colorado, USA) connected to a Teflon tube following European Respiratory Task Force recommendations, ${ }^{21}$ as previously described by our group. ${ }^{22}$

\section{Sputum sampling and preparation}

Sputum was induced and processed following standard methodology. ${ }^{23}$ Briefly, sputum was incubated with four times its weight of $0.01 \mathrm{M}$ dithiothreitol in Hank's buffered salt solution (HBSS) at $4^{\circ} \mathrm{C}$ for $15 \mathrm{~min}$. Sputum dilution was proportional to the collected weight, thus standardising between time points and patients the supernatants used for assay of proteinases and other markers. The volume of HBSS was doubled (tenfold dilution of original sputum sample) and incubated for a further $5 \mathrm{~min}$. The suspension was then filtered through $50 \mu \mathrm{m}$ nylon gauze to remove mucus and debris without removing any of the cells and centrifuged at $790 \mathrm{~g}$ for $10 \mathrm{~min}$. The cell-free supernatant was removed and stored at $-70^{\circ} \mathrm{C}$. The cell pellet was resuspended. Total cell count was determined with a Neubauer haemocytometer. The trypan blue exclusion method was used to determine cell viability. Cytospins were made from the cell suspension and stained with Diff-Quik to obtain a differential cell count. Sputum samples with less than a million cells were considered inadequate and were therefore excluded from analysis. Cells were incubated in a six-well plate (multiwell primaria surface modified polystyrene, Falcon 35.3846) at a density of $1 \times 10^{6}$ cells/well in $2 \mathrm{ml}$ of medium (RPMI 1640, $10 \%$ FCS, L-Glu). After $4 \mathrm{~h}$, supernatant was removed and macrophages were scraped from the plate and lysed for protein extraction.

\section{Cytokine determination}

The concentration of cytokines (TNF $\alpha$, IL6 and IL8) in sputum supernatant was determined by flow cytometry (CBA, Human Inflammation Kit, BD Biosciences, San Jose, California, USA) following the manufacturer's instructions. Assay sensitivity, as expressed by the manufacturer, were as follows: $3.6 \mathrm{pg} / \mathrm{ml}$ for IL8, $2.5 \mathrm{pg} / \mathrm{ml}$ for IL6 and $3.7 \mathrm{pg} / \mathrm{ml}$ for TNF $\alpha$.

\section{Total antioxidant status (TAS)}

TAS in sputum was measured by a colorimetric test (Randox Laboratories, Crumlin, UK) as previously described by our group. $^{24}$

\section{HDAC activity}

HDAC activity of sputum macrophage nuclear extracts was measured with a non-isotopic assay using a fluorescent derivative of epsilon-acetyl lysine (HDAC fluorescent Activity Assay Kit, BIOMOL, Plymouth, Pennsylvania, USA), as previously described. ${ }^{17}$

\section{NF-кB activity}

$N F-\kappa B$ activation was assessed in sputum macrophage nuclear extracts using the TransAM NF- $\mathrm{B}$ p65 Transcription Factor Assay Kit (Active Motif, Carlsbad, California, USA), as previously described. ${ }^{25}$

\section{Statistical analysis}

Results are expressed as mean (SEM) values or median (range) values in non-normally distributed variables. HDAC activity, NF- $\kappa \mathrm{B}$ activation values, IL8, IL6, TNF $\alpha$, eNO, TAS and $\mathrm{FEV}_{1}$ values were normally distributed and were compared between groups using two-way ANOVA. Analyses were performed for the intention-to-treat population. A $p$ value of $<0.05$ (twotailed) was considered statistically significant. Analysis was

Table 1 Demographic and clinical characteristics of patients with COPD at the time of admission

\begin{tabular}{|c|c|c|c|}
\hline & $\begin{array}{l}\text { All } \\
(n=35)\end{array}$ & $\begin{array}{l}\text { Theophylline } \\
(\mathrm{n}=16)\end{array}$ & $\begin{array}{l}\text { Standard therapy } \\
(\mathrm{n}=19)\end{array}$ \\
\hline Age (years) & $67.6(1.3)$ & $66.7(1.7)$ & $68.2(2.1)$ \\
\hline Gender (M/F) & $35 / 0$ & $16 / 0$ & $19 / 0$ \\
\hline Smoking (pack-years) & $58.6(4.3)$ & $62.9(6.9)$ & $54.1(6.4)$ \\
\hline Active smoking, $\mathrm{n}(\%)$ & $15(42 \%)$ & $8(50 \%)$ & $7(37 \%)$ \\
\hline Charlson index & $1.83(0.2)$ & $1.77(0.3)$ & $1.88(0.2)$ \\
\hline $\mathrm{FEV}_{1}(\mathrm{I})$ & $1.03(0.1)$ & $0.99(0.1)$ & $1.11(0.1)$ \\
\hline $\mathrm{FEV}_{1}(\%)$ & $38.4(2.1)$ & $39.2(3.8)$ & $37.7(1.9)$ \\
\hline $\mathrm{FEV}_{1} / \mathrm{FVC}(\%)$ & $46.39(1.9)$ & $45.34(3.3)$ & $47.89(2.7)$ \\
\hline $\begin{array}{l}\text { Salbutamol } \\
\text { reversibility (\%) }\end{array}$ & $5.17(1.3)$ & $7.33(1.6)$ & $3.89(1.6)$ \\
\hline ICS, n (\%) & $21(60 \%)$ & $11(68 \%)$ & $10(53 \%)$ \\
\hline $\mathrm{PaO}_{2}(\mathrm{~mm} \mathrm{Hg})$ & $56.4(1.6)$ & $53.7(2.4)$ & $58.6(1.7)$ \\
\hline $\mathrm{PaCO}_{2}(\mathrm{~mm} \mathrm{Hg})$ & $45.6(1.7)$ & $48.4(2.5)$ & $42.7(2.2)$ \\
\hline
\end{tabular}

Values are mean (SEM) unless indicated otherwise.

$\mathrm{FEV}_{1}$, forced expiratory volume in $1 \mathrm{~s}$; FVC, forced vital capacity; ICS, inhaled corticosteroids; $\mathrm{PaO}_{2}, \mathrm{PaCO}_{2}$, arterial oxygen and carbon dioxide tensions. 


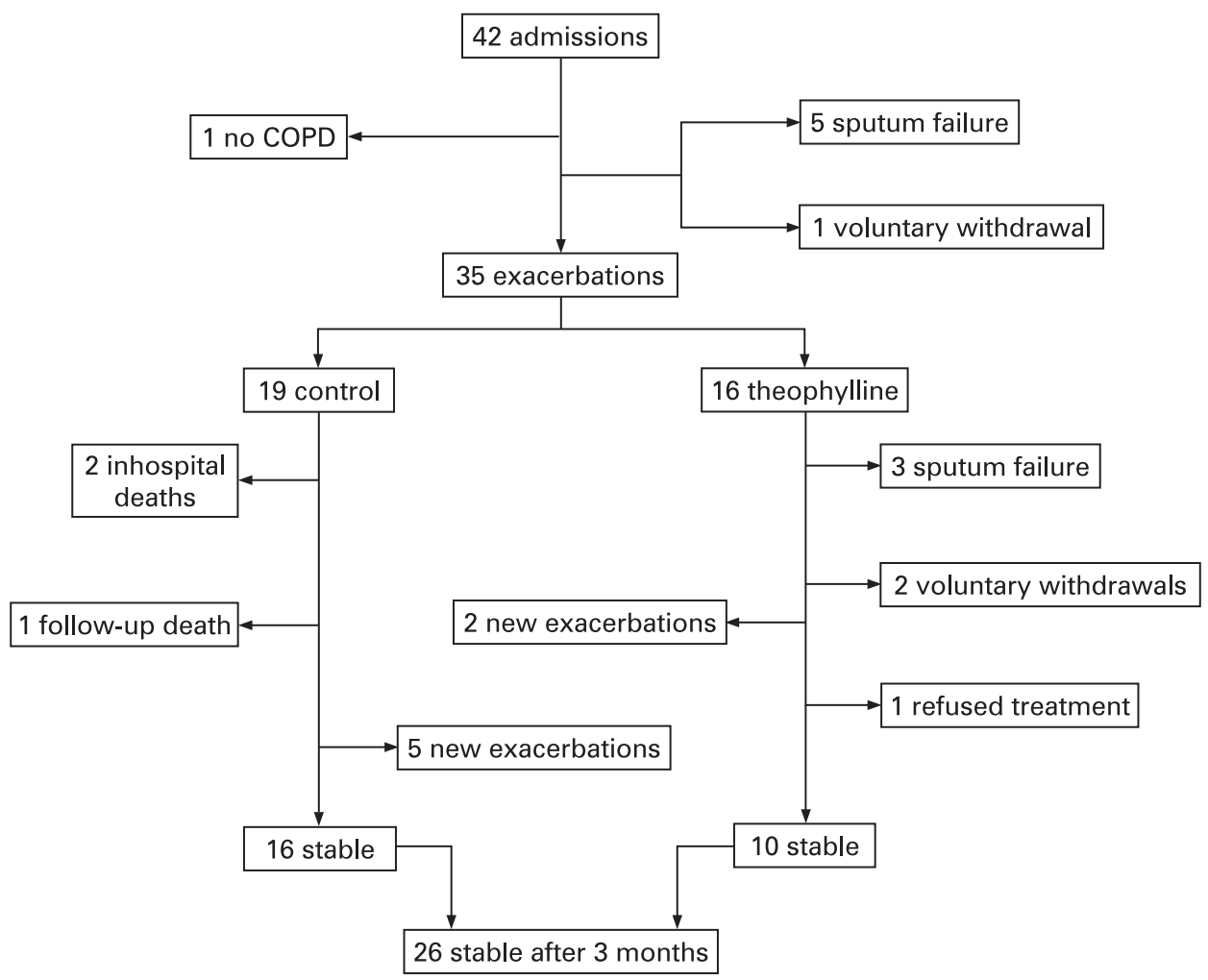

Figure 1 Flow diagram of study participants from recruitment into the two arms to outcomes. COPD, chronic obstructive pulmonary disease.

performed using GraphPad Prism software (GraphPad Software, San Diego, California, USA).

\section{RESULTS \\ Clinical data}

Of the 42 patients originally recruited, 7 were excluded within the first $24 \mathrm{~h}$ because of an inadequate sputum sample $(n=5)$, absence of airway obstruction in the first spirometry $(n=1)$ and voluntary study withdrawal $(n=1)$. Except for the patient excluded with post-bronchodilator $\mathrm{FEV}_{1} / \mathrm{FVC}$ of $78 \%$, the other 6 patients were not statistically different from the patients included in the study. The remaining 35 patients (all male) entered the study and were randomised to receive standard treatment (19 patients) or standard treatment plus oral theophylline $100 \mathrm{mg}$ twice daily (16 patients). Table 1 shows the baseline demographic, clinical and functional characteristics of all participants. In the theophylline group, 10 patients were receiving treatment with a combination of inhaled corticosteroids and long-acting $\beta$ agonist and 6 were receiving tiotropium, whereas in the control group this was the case in 11 and 9 patients, respectively. The history of previous exacerbations revealed that, in the theophylline group, 3 patients had had $\geqslant 2$ exacerbations and 3 had had 1 exacerbation in the previous year, whereas in the control group 2 patients had had $\geqslant 2$ exacerbations and 5 had had 1 exacerbation in the previous year.

There were no differences in basic and liver function laboratory parameters between the two groups at the time of admission. All patients were treated with systemic corticosteroids which were tapered during the admission. The dose and duration of treatment was not standardised, and it was individualised upon symptoms and physical examination. All patients had a prescription of oral prednisone at discharge. Median total dose was $590 \mathrm{mg}$ (range 400-920) and median total time of corticosteroid treatment was 18 days (15-30) in the theophylline group and $600 \mathrm{mg}$ (425-1000) and 19 days (15-32) in the standard therapy group. These figures were not statistically different. Patients in the theophylline group were treated during a median time of 102 days (range 94-140). Compliance was assessed by measuring theophylline levels at the end of the study. Of the 35 patients studied, 27 were treated with antibiotics (12 in the theophylline group and 15 in the standard therapy group, $p=0.15)$. The antibiotics given were amoxiclav in 19 patients, levofloxacin in 6 patients and trimethoprim/sulfamethoxazole in 2 patients.

Four patients in the theophylline group and 3 in the control group had a positive sputum culture taken at the time of admission. The bacteria isolated were Pseudomonas aeruginosa (2), Stenotrophomona sp (1), Haemophilus influenzae (2), Streptococcus pneumoniae (1) and Proteus mirabilis (1). Two patients died during hospitalisation, both in the standard treatment arm. The length of hospital stay was not different between the two groups (6.31 (0.27) days in the theophylline group vs 7.35 (1.05) days in the group receiving standard therapy, $p=0.38)$. After discharge, 2 patients in the theophylline group (12.5\%) and 5 in the standard therapy group (26\%) had a new ECOPD. Theophylline was maintained during the new admission and both patients received systemic steroids and antibiotics (one amoxiclav and the other levofloxacin) and were discharged after 7 and 10 days of admission, respectively. The median time to a new exacerbation was 36 days (range 32-40) in the theophylline group and 32 days (range 12-46) in the control group. There was an additional death in the standard therapy group during follow-up. Some patients were lost during follow-up owing to unwillingness to participate $(n=2)$, discontinuation of theophylline because of side effects $(n=1)$ or an inadequate sputum sample $(n=3)$. As a result, 16 of the 19 patients in the standard treatment arm and 10 of the 16 in the theophylline arm were studied again when clinically stable 

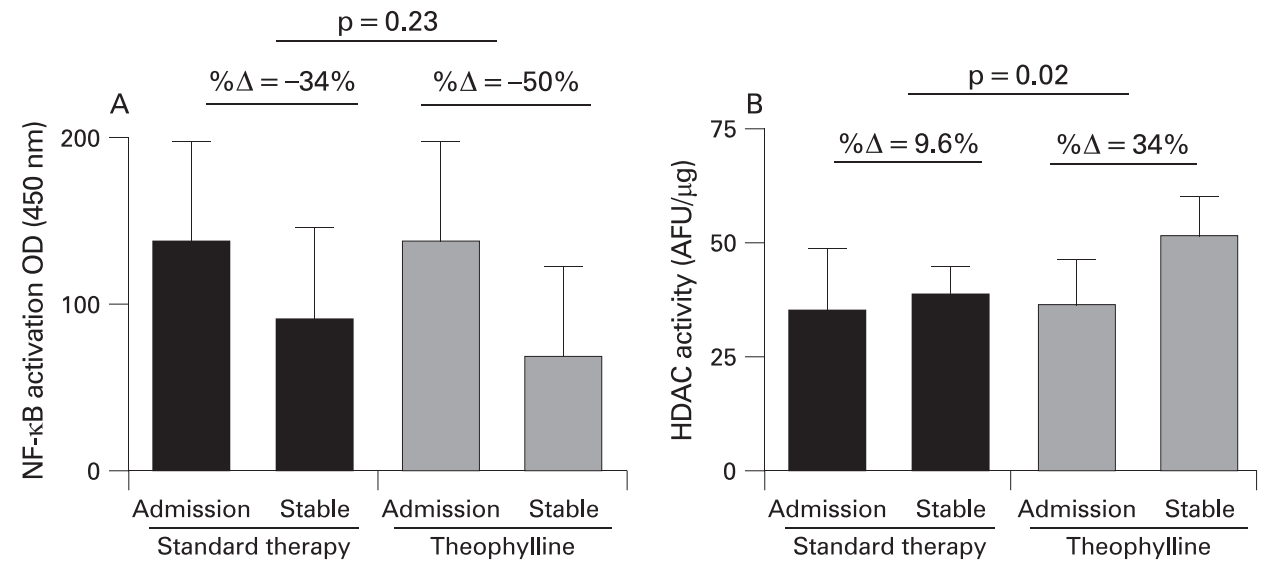

Figure 2 Mean (SEM) values of (A) nuclear factor- $\kappa B(N F-\kappa B)$ and $(B)$ histone deacetylase (HDAC) activity in patients with an exacerbation of chronic obstructive pulmonary disease before and 3 months after receiving standard therapy with or without low-dose theophylline; $p$ values refer to comparisons between groups.

(fig 1). Compared with measurements obtained in the emergency room, $\mathrm{FEV}_{1}$ improved by $24 \%$ ( $95 \%$ confidence interval (CI) $2 \%$ to $45 \%$ ) during clinical stability in patients receiving standard therapy and by $37 \%$ (95\% CI $1 \%$ to $63 \%$ ) in those receiving theophylline in addition $(p<0.01$ between groups, mean differences -0.401 vs -0.251 ).

\section{Anti-inflammatory effects of standard therapy}

NF- $\kappa \mathrm{B}$ activation was reduced during recovery of ECOPD by $34 \%$ (95\% CI $23.8 \%$ to $44 \%$ ) compared with exacerbation in the group receiving standard therapy (fig $2 \mathrm{~A}$ ). In parallel, there was a statistically significant reduction in the eNO concentration and in the sputum levels of TNF $\alpha$, IL6 and IL8 (fig 3). Sputum TAS increased during recovery compared with exacerbation by $70 \% \quad(95 \%$ CI $-4.0 \%$ to $145 \% ; 0.55 \quad(0.1) \mathrm{mmol} / \mathrm{l}$ vs 0.95 (0.2) $\mathrm{mmol} / \mathrm{l}$ ), although HDAC activity did not change (fig $2 \mathrm{~B}$ ).

\section{Effects of theophylline}

Mean (SEM) plasma levels of theophylline during follow-up were $4.6(1.2) \mathrm{mg} / \mathrm{l}$. Two patients receiving theophylline experienced mild nausea and two reported headache. The reduction in NF- $\kappa \mathrm{B}$ activation or the increase in TAS seen in the standard therapy group were not influenced by the addition of low-dose theophylline (fig 2A). TAS in sputum supernatant increased by $105.9 \%$ (95\% CI $56 \%$ to $155 \%$; 0.37 (0.1) $\mathrm{mmol} / \mathrm{l}$ vs $0.69(0.2) \mathrm{mmol} / \mathrm{l})$ from exacerbation to stable phase, but no statistical differences with the standard therapy group were found (see above). By contrast, the addition of low-dose theophylline resulted in a statistically significant increase in HDAC activity (fig 2B) compared with the standard therapy group that was accompanied by further reductions in the sputum concentrations of TNF $\alpha$ and IL8 (fig 3).

\section{DISCUSSION}

This study shows that low-dose theophylline enhances the activity of HDAC and the anti-inflammatory effects of steroids in male patients with COPD with sputum production hospitalised because of an exacerbation of the disease.

\section{Previous studies}

We have previously shown that HDAC activity is reduced in patients with stable COPD, that this contributes to steroid resistance, $^{23}$ and that both HDAC activity $^{15}$ and steroid sensitivity ${ }^{17}$ can be restored by low-dose theophylline. The present study extends these previous observations for the first time to patients hospitalised because of an acute exacerbation of the disease (ECOPD).

Oxidative stress reduces HDAC activity in patients with stable COPD. ${ }^{11}$ This is associated with histone-4 acetylation of the IL8 promoter which in turn increases IL8 transcription, ${ }^{3}$ and with enhanced NF- $\kappa B$-mediated gene expression. ${ }^{25}$ Increased IL8 synthesis contributes to neutrophil recruitment, a key pathogenic event in COPD. ${ }^{26}$ Oxidative stress is known to increase during ECOPD, ${ }^{7}$ but its potential effects on HDAC activity have not previously been investigated in this setting.

The anti-inflammatory effects of theophylline have been previously described, and the proposed mechanisms of action vary from phosphodiesterase inhibition ${ }^{27}$ to mediator inhibition, ${ }^{28}$ increased apoptosis ${ }^{29}$ or inhibition of NF- $\kappa B .{ }^{30}$ These direct anti-inflammatory effects, however, occur at statistically significant higher concentrations of theophylline than those used in clinical practice $(>20 \mathrm{mg} / \mathrm{l})$. In contrast, at low doses theophylline increases HDAC activity, ${ }^{17}$ reduces the neutrophil concentration in sputum ${ }^{31}$ and large airways $s^{32}$ and enhances the anti-inflammatory effects of glucocorticoids. ${ }^{15}$ These biological effects of low-dose theophylline have been described after longterm treatment, ${ }^{33}$ but can be seen after just 4 weeks of treatment. $^{34}$ They have not been investigated previously in patients hospitalised because of ECOPD.

\section{Interpretation of novel findings}

We found that NF- $\mathrm{B}$ activation and the concentration of several inflammatory markers in sputum decreased from COPD exacerbation to clinical stability, and that the addition of lowdose theophylline decreased the concentration of IL8 and TNF $\alpha$ even further. We propose that these differential effects may be due to a specific effect of theophylline on HDAC activity for these two particular genes (figs 2 and 3 ).

The effect of theophylline on HDAC activity can be explained by different mechanisms. First, oxidative stress causes HDAC dysfunction ${ }^{11}$ and, in keeping with previous studies, ${ }^{67}$ we found increased oxidative stress during ECOPD as indicated by reduced TAS. However, it is unlikely that this contributes to the differences observed in patients receiving theophylline because TAS recovery was similar in both groups, with no statistical differences between them. Second, increased nitrative 

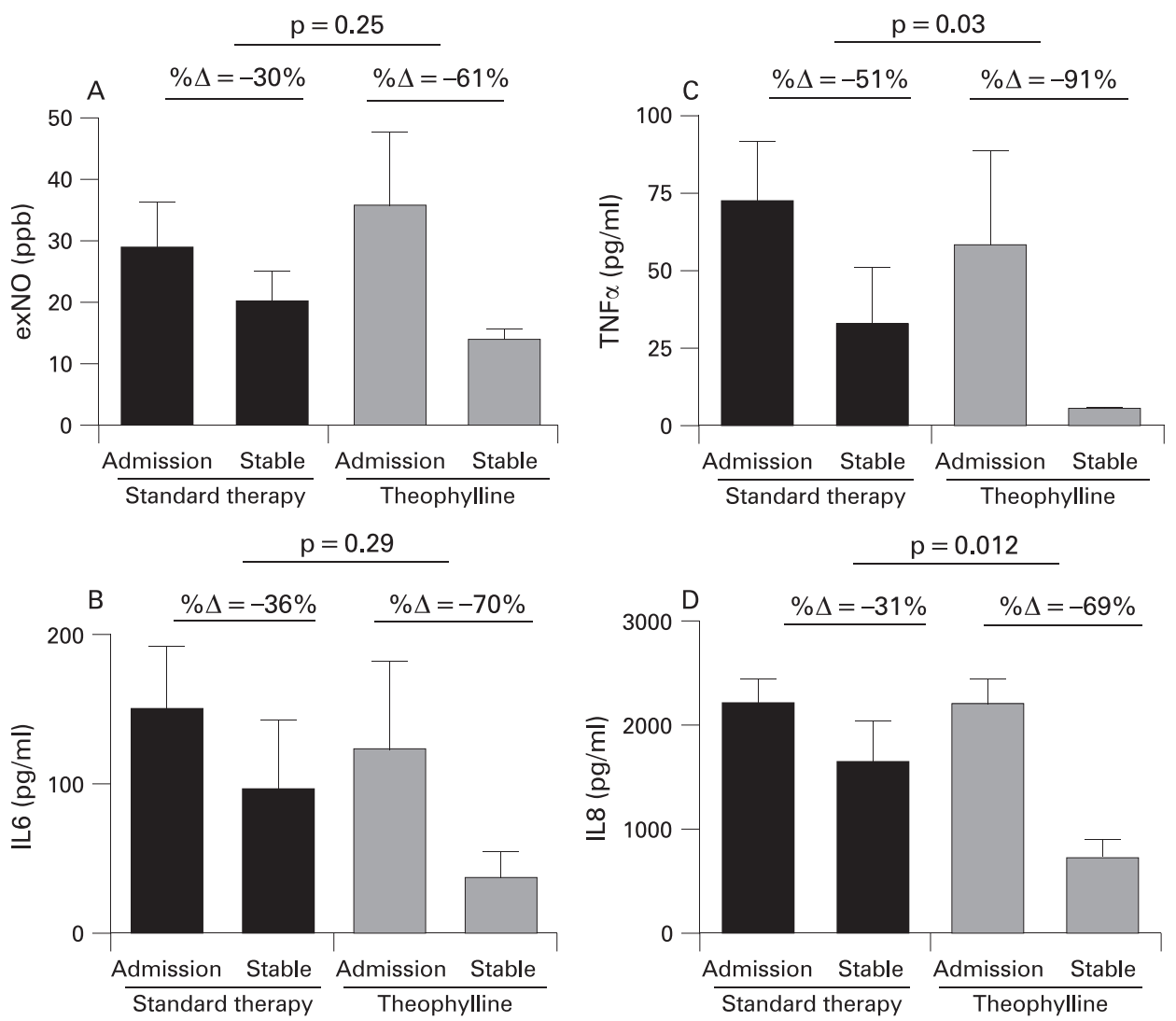

Figure 3 Mean (SEM) levels of (A) nitric oxide concentration in exhaled air (eNO), (B) interleukin-6 (IL6), (C) tumour necrosis factor $\alpha$ (TNF $\alpha$ ) and (D) interleukin-8 (IL8) in sputum supernatants in patients with an exacerbation of chronic obstructive pulmonary disease before and after receiving standard therapy with or without low-dose theophylline; $p$ values refer to comparisons between groups.

stress may generate peroxynitrite which nitrates tyrosine residues on HDAC2 and impairs enzyme activity. ${ }^{12}$ As previously shown, ${ }^{22}$ we found that eNO concentration, an indirect marker of nitrative stress, increased during ECOPD (fig 3A). Interestingly, however, eNO recovery during clinical stability showed a trend, although not statistically significant, to be enhanced in patients receiving theophylline (fig 3A), suggesting that reduced nitrative stress may contribute to enhanced HDAC activity (fig 2A). This would be in keeping with previous studies showing that theophylline reduces nitrative stress in vitro and ex vivo. ${ }^{32}$

By comparing the exacerbation with the stable phase 3 months later, the effect of low-dose theophylline on HDAC activity would not only affect the inflammatory mechanisms during exacerbations but also it would have an effect on chronic baseline inflammation that characterises COPD. However, the reduction in NF- $\kappa \mathrm{B}$ activation seen at stability in both groups indicates that the burden of inflammation is different from the exacerbation to the stable phase, and the fact that HDAC activity is not reduced at exacerbation compared with the stable phase of COPD in the control group suggests that HDAC activity is not able to be modified by corticosteroids alone, which confirms previous observations. ${ }^{23}$

Irrespective of the underlying molecular mechanism explaining enhanced HDAC activity induced by theophylline, it is well established that the latter can improve steroid sensitivity in COPD. ${ }^{17}$ This would explain the additional reduction in the inflammatory markers observed in patients receiving low-dose theophylline in addition to standard therapy, which includes treatment with steroids (fig 3).

\section{Potential limitations}

This was a prospective randomised single-blind study, but it was not placebo-controlled. The primary goal of the study was to investigate the biological effects of low-dose theophylline on HDAC activity and inflammation in patients with ECOPD, and not to establish its potential clinical usefulness-something which would have been limited anyway by the relatively small sample size used in the study. An additional limitation was that our population was entirely male and we had to exclude patients with inadequate sputum samples, so we could not generalise the findings to all COPD exacerbations. Interestingly, however, despite these limitations, we observed that patients treated with low-dose theophylline in addition to the recommended treatment for ECOPD had better clinical outcomes, such as FEV 1 recovery. Mortality was also decreased in this group, but this effect was not statistically different between the groups. However, these observations-in combination with the clearcut molecular effects described here-provide a strong rationale for investigating the potential clinical relevance of this therapeutic strategy in a large randomised, double-blind, placebo controlled trial.

In conclusion, this study demonstrates that low-dose theophylline increases HDAC activity and further reduces inflammation when added to the standard therapeutic regime of ECOPD. The clinical implications of these molecular observations deserve investigation in a larger double blind, placebo controlled, randomised clinical trial.

Acknowledgements: The authors thank Dr Joan Vidal, Dr Javier Garcia and Dr Jordi Puiguriguer from the A\&E department for their cooperation during recruitment, Dr Alberto Fuster for his valuable help in the total antioxidant status assay, Dr Catalina 
Crespi for her assistance with the cytometric bead aArray and Ms Meritxell Arques for her contribution to designing the database. Ciberes is an initiative of the Instituto de Salud Carlos III.

Funding: Supported in part by FIS 04/2146, ABEMAR, SEPAR and Medical Research Council (UK).

Competing interests: BGC has received lecture fees and has served on Scientific Advisory Boards for GlaxoSmithKline (GSK). AstraZeneca and Novartis. KI is an employee of RespiVert Ltd. PB has received research funding, lecture fees and has served on Scientific Advisory Boards for GSK, AstraZeneca, Boehringer-Ingelheim, Novartis, Altana and Pfizer, all of whom have an interest in new therapies for COPD. AA has received research funding from AstraZeneca, Pfizer and Boehringer Ingelheim and lecture fees for speaking at conferences sponsored by GSK, AstraZeneca and Almirall during the past five years. He has also served on advisory boards for GSK, Almirall and Altana. The other authors have no competing interests with this paper.

Ethics approval: All patients were informed of the nature and purpose of the study and gave their written consent. The study was approved by the local ethics committee.

\section{REFERENCES}

1. Rabe KF, Hurd S, AnzuetoA, et al. Global strategy for the diagnosis, management, and prevention of chronic obstructive pulmonary disease: GOLD executive summary. Am J Respir Crit Care Med 2007;176:532-55.

2. Barnes PJ, Ito K, Adcock IM. Corticosteroid resistance in chronic obstructive pulmonary disease: inactivation of histone deacetylase. Lancet 2004;363:731-3.

3. Ito $\mathbf{K}$, Ito $\mathrm{M}$, Elliott WM, et al. Decreased histone deacetylase activity in chronic obstructive pulmonary disease. N Engl J Med 2005;352:1967-76.

4. Caramori G, Romagnoli M, Casolari $\mathrm{P}$, et al. Nuclear localisation of p65 in sputum macrophages but not in sputum neutrophils during COPD exacerbations. Thorax 2003; 58:348-51.

5. Barnes PJ, Karin M. Nuclear factor-kappaB: a pivotal transcription factor in chronic inflammatory diseases. N Engl J Med 1997;336:1066-71.

6. Rahman I, Morrison D, Donaldson K, et al. Systemic oxidative stress in asthma, COPD, and smokers. Am J Respir Crit Care Med 1996;154:1055-60.

7. Drost EM, Skwarski KM, Sauleda J, et al. Oxidative stress and airway inflammation in severe exacerbations of COPD. Thorax 2005;60:293-300.

8. Donaldson GC, Wedzicha JA. COPD exacerbations.1: Epidemiology. Thorax 2006;61:164-8.

9. Pinto-Plata VM, Livnat G, Girish M, et al. Systemic cytokines, clinical and physiological changes in patients hospitalized for exacerbation of COPD. Chest 2007:131:37-43.

10. Hurst JR, Perera WR, Wilkinson TM, et al. Exacerbation of chronic obstructive pulmonary disease: pan-airway and systemic inflammatory indices. Proc Am Thorac Soc 2006;3:481-2.

11. Adcock IM, Cosio B, Tsaprouni L, et al. Redox regulation of histone deacetylases and glucocorticoid-mediated inhibition of the inflammatory response. Antioxid Redox Signal 2005; 7:144-52.

12. Tomita K, Barnes PJ, Adcock IM. The effect of oxidative stress on histone acetylation and IL-8 release. Biochem Biophys Res Commun 2003;301:572-7.

13. Barnes PJ. Theophylline: new perspectives for an old drug. Am J Respir Crit Care Med 2003;167:813-8.
14. Barnes PJ. Theophylline for COPD. Thorax 2006;61:742-4.

15. Ito K, Lim S, Caramori G, et al. A molecular mechanism of action of theophylline: induction of histone deacetylase activity to decrease inflammatory gene expression. Proc Natl Acad Sci U S A 2002;99:8921-6.

16. Zhou $Y$, Wang $X$, Zeng $X$, et al. Positive benefits of theophylline in a randomized, double-blind, parallel-group, placebo-controlled study of low-dose, slow-release theophylline in the treatment of COPD for 1 year. Respirology 2006;11:603-10.

17. Cosio BG, Tsaprouni L, Ito K, et al. Theophylline restores histone deacetylase activity and steroid responses in COPD macrophages. J Exp Med 2004;200:689-95.

18. Anthonisen NR, Manfreda J, Warren CP, et al. Antibiotic therapy in exacerbations of chronic obstructive pulmonary disease. Ann Intern Med 1987;106:196-204.

19. American Thoracic Society. Standardization of spirometry, 1994 update. Am J Respir Crit Care Med 1995;152:1107-36.

20. Roca J, Sanchis J, gusti-Vidal A, et al. Spirometric reference values from a Mediterranean population. Bull Eur Physiopathol Respir 1986;22:217-24.

21. Kharitonov S, Alving K, Barnes PJ. Exhaled and nasal nitric oxide measurements: recommendations. The European Respiratory Society Task Force. Eur Respir J 1997; 10:1683-93

22. Agusti AG, Villaverde JM, Togores B, et al. Serial measurements of exhaled nitric oxide during exacerbations of chronic obstructive pulmonary disease. Eur Respir $J$ 1999;14:523-8.

23. Keatings VM, Collins PD, Scott DM, et al. Differences in interleukin-8 and tumor necrosis factor-alpha in induced sputum from patients with chronic obstructive pulmonary disease or asthma. Am J Respir Crit Care Med 1996;153:530-4.

24. Barcelo A, Barbe F, de la PM, et al. Antioxidant status in patients with sleep apnoea and impact of continuous positive airway pressure treatment. Eur Respir $\mathrm{J}$ 2006;27:756-60.

25. Ito K, Yamamura S, Essilfie-Quaye $\mathrm{S}$, et al. Histone deacetylase 2-mediated deacetylation of the glucocorticoid receptor enables NF-kappaB suppression. J Exp Med 2006;203:7-13.

26. Barnes PJ. Chronic obstructive pulmonary disease. N Engl J Med 2000;343:269-80

27. Rabe KF, Magnussen H, Dent G. Theophylline and selective PDE inhibitors as bronchodilators and smooth muscle relaxants. Eur Respir J 1995;8:637-42.

28. Mascali JJ, Cvietusa P, Negri J, et al. Anti-inflammatory effects of theophylline: modulation of cytokine production. Ann Allergy Asthma Immunol 1996;77:34-8.

29. Yasui K, Hu B, Nakazawa T, et al. Theophylline accelerates human granulocyte apoptosis not via phosphodiesterase inhibition. J Clin Invest 1997;100:1677-84.

30. Tomita K, Chikumi H, Tokuyasu H, et al. Functional assay of NF-kappaB translocation into nuclei by laser scanning cytometry: inhibitory effect by dexamethasone or theophylline. Naunyn Schmiedebergs Arch Pharmacol 1999;359:249-55.

31. Culpitt SV, De Matos C, Russell RE, et al. Effect of theophylline on induced sputum inflammatory indices and neutrophil chemotaxis in chronic obstructive pulmonary disease. Am J Respir Crit Care Med 2002;165:1371-6.

32. Hirano $\mathbf{T}$, Yamagata $\mathrm{T}$, Gohda $\mathrm{M}$, et al. Inhibition of reactive nitrogen species production in COPD airways: comparison of inhaled corticosteroid and oral theophylline. Thorax 2006;61:761-6.

33. liboshi H, Ashitani J, Katoh S, et al. Long-term treatment with theophylline reduces neutrophils, interleukin-8 and tumor necrosis factor-alpha in the sputum of patients with chronic obstructive pulmonary disease. Pulm Pharmacol Ther 2007:20:46-51.

34. Kobayashi M, Nasuhara $Y$, Betsuyaku T, et al. Effect of low-dose theophylline on airway inflammation in COPD. Respirology 2004:9:249-54.

\section{Access the latest content chosen by our Editors}

BMJ Journals editors select an article from each issue to be made free online immediately on publication. Other material is free after 12 months to non-subscribers. Access the Editor's Choice from the home page- or expand your horizons and see what the other BMJ Journals editors have chosen by following the links on any BMJ Journal home page. 